\title{
Lymphopenia as an early predictor of disease severity in COVID-19
}

Shaheen Ali Khan, Laraib Saeed, Muhammad Naveed Anwar, Wajeeha Qayyum, Zuhayr Ahmad Mufti, Sofia Haider Durrani

\section{Submitted}

September 10, 2020

Accepted

September 28, 2020

Author Information

Dr. Shaheen Ali Khan

Trainee Medical Officer,

Medicine and Allied, Rehman

Medical Institute, Peshawar,

KP, Pakistan.

(Corresponding Author)

Email:

drshaheenalikhan@gmail.com

Dr. Muhammad Naveed

Anwar

Consultant Gastroenterologist

Rehman Medical Institute,

Peshawar, KP, Pakistan

Dr. Laraib Saeed

Trainee Medical Officer,

Medicine and Allied,

Rehman Medical Institute,

Peshawar, KP, Pakistan

Dr. Wajeeha Qayyum

Senior Registrar

Medicine and Allied

Rehman Medical Institute,

Peshawar, KP, Pakistan.

Dr. Zuhayr Ahmad Mufti Trainee Medical Officer,

General Medicine

Hayatabad Medical Complex,

Peshawar, KP, Pakistan

Dr. Sofia Haider Durrani

Assistant Professor

Oral Pathology

Sardar Begum Dental

College, Peshawar, KP,

Pakistan.

Citation: Khan SA, Saeed L, Qayyum W, Mufti ZA,

Durrani SH. Lymphopenia as an early predictor of disease severity in COVID-19. J

Rehman Med Inst. 2020 Jul-

Sep;6(3):8-11.

\section{ABSTRACT}

Introduction: Research has implicated an exaggerated or self-directed immune response as a factor in determining patient outcome in COVID-19. Initial reports identify lymphopenia as having a predictive role in COVID-19 related disease severity.

Objective: To document the occurrence of lymphopenia in COVID-19 patients and explore its association with demographic factors and disease course.

Materials \& Methods: This cross sectional, observational study was conducted at Rehman Medical Institute, Peshawar from May, 2020 to August, 2020. All patients admitted to the hospital with COVID-19 diagnosis were included. Relevant information including demographics, disease severity, laboratory findings \& outcomes were noted in a predesigned Performa. The data were analyzed on SPSS 22 for descriptive and comparative statistics; $\mathrm{p} \leq 0.05$ denoted significance.

Results: A total of 216 patients were included, of whom $172(79.6 \%)$ were males. The mean age was $54.61 \pm$ 14.35 years. Classic lymphopenia was found in 67 (31.5\%), 66 (31.0\%) had borderline lymphopenia, and $80(37.6 \%)$ had no lymphopenia. Of the classic lymphopenia group, 14 (20.9\%) had mild disease, 21 $(31.3 \%)$ had moderate disease, 18 (26.9\%) had severe disease \& $14(20.9 \%)$ had critical disease. In patients with borderline lymphopenia $31(47.0 \%)$ had mild disease, $17(25.8 \%)$ moderate disease, 14 (21.2\%) had severe disease and $04(6.1 \%)$ had critical disease. In patients with no lymphopenia, $32(40.0 \%)$ had mild, 32 (40.0\%) had moderate, $14(17.5 \%)$ had severe \& 02 $(2.5 \%)$ had critical disease. Mortality associated with classical lymphopenia was $37.3 \%(n=25)$ whereas $43.3 \%$ $(n=29)$ patients recovered. Male gender, disease severity and outcome were statistically linked to lymphopenia; no statistical significance was observed with age.

Conclusion: A statistically significant association of lymphopenia with severe/critical disease and adverse outcomes was noted.

\section{Keywords: Lymphopenia, COVID-19, Pandemic.}

The authors declared no conflict of interest. All authors contributed substantially to the planning of research, data collection, data analysis, and write-up of the article, and agreed to be accountable for all aspects of the work.

\section{INTRODUCTION}

In December 2019, a group of patients in Wuhan, China, were found suffering from a mysterious respiratory illness of undetermined etiology. All the patients were found to have been exposed to a wet animal market in Wuhan. ${ }^{1}$ The causative agent was later identified as a new coronavirus eventually named severe acute respiratory syndrome coronavirus 2 (SARS-COV-2) and the disease it causes was labelled COVID-19. Coronaviruses are RNA viruses that can infect mammals, including humans and birds and lead to heterogeneous diseases that can involve the central nervous, respiratory \& gastrointestinal systems. Two of the six coronaviruses that can infect humans, have led to fatal outbreaks of diseases such as the Severe Acute Respiratory Syndrome outbreaks in 2002 and 2003 in China \& severe respiratory disease outbreaks in 2012 in the Middle East respectively. ${ }^{2}$

As of August 1, 2020 there have been 17.2 million reported cases of COVID-19 worldwide with 67 thousand reported deaths. Pakistan has a reported 278 thousand cases and 5951 deaths. The cause of the low mortality is thought to be under reporting. ${ }^{3}$

COVID-19 shows a whole rainbow of symptoms ranging from mild to critical with $81 \%$ of patients exhibiting mild and 5\% exhibiting critical disease. Common lab aberrations seen are lymphopenia, ESR and inflammatory marker elevations, particularly C-reactive protein, lactate dehydrogenase, ferritin and d-dimers. ${ }^{4-6}$.

Lymphocytes play a pivotal role in immunity and inflammatory reaction to foreign invaders within the body. ${ }^{7}$ A prominent functional decline in $\mathrm{T}$ Lymphocytes is reported in COVID-19. ${ }^{8}$

Low Lymphocyte counts have been correlated with disease severity and the patients that died reportedly had significantly fewer lymphocytes compared to survivors. Four possible mechanisms have been postulated to explain lymphocyte deficiencies in COVID-19: First, a direct cytotoxic effect of the virus on lymphocytes that express surface ACE2 receptors whereby viral entry and eventual destruction of the cell occurs. Second, the virus might directly destroy lymphatic organs such 
as the thymus and spleen. Third, inflammatory cytokines, such as TNF-alpha and IL-6, released during the infection may lead to lymphocyte apoptosis. Fourth, inhibition of lymphocytes by an acidotic hyperlactemic environment induced within the body. The elevated blood lactic acid levels, seen in patients with severe COVID-19 are thought to depress the growth and division of lymphocytes. These various mechanisms are thought to work together in unison to lead to lymphopenia, however further research into these postulated mechanisms is required. ${ }^{7}$

Timely identification of patients who may quickly progress to critical disease is very important in the management of COVID19 , with the aim to prioritize care and direct valuable resources where needed the most, as health care systems everywhere and particularly in low to middle income countries like Pakistan are under immense strain. ${ }^{8}$ Therefore it is essential to find an economical, easily available \& accurate measure for quick identification of potential patients that can progress to severe or critical disease, so as to prioritize their management. Lymphocyte count may just be the biomarker we are looking for. However evidence regarding the extrapolative role of lymphocyte count in predicting the severity of COVID-19 is still limited. Herein, we analyze data collected from COVID-19 patients to determine the possible role of lymphocyte count in identifying patients at risk of severe disease early on in order to prioritize their management.

The objective of the study was to document the occurrence of lymphopenia in COVID-19 patients, and to explore its association with demographic factors and disease course.

\section{MATERIALS \& METHODS}

This cross sectional descriptive study was conducted at Rehman Medical Institute, Peshawar, Khyber Pakhtunkhwa, Pakistan from May 2020 to August 2020, on all patients admitted to the hospital that tested positive for COVID-19 on an RT-PCR performed on nasopharyngeal secretions. ${ }^{9}$ Approval was obtained from the Research Ethics Committee of Rehman Medical Institute. The requirement for written, informed consent was fulfilled by either the patients or their representatives in case patients were incapacitated. In the instance of a negative RT-PCR but high clinical suspicion, an HRCT was done and classic findings in accordance with the guidelines set by the American Radiology Association ARA were taken as being diagnostic. ${ }^{10}$

Recent studies on COVID-19 have defined lymphopenia as a lymphocyte count $\leq 1-1.5 \times 10^{9} / \mathrm{L}$. Four recent studies defined it as $\leq 1.1 \times 10^{9} / \mathrm{L}$ and one as $\leq 1.5 \times 10^{9} / \mathrm{L} .{ }^{8}$ For the purpose of precision we divided our patients into three groups: Group 1 (Classic Lymphopenia) contained patients with lymphocyte counts $\leq 1 \times 10^{9} / \mathrm{L}$, Group 2 (Borderline Lymphopenia) had patients with lymphocyte counts of $1-1.5 \times 10^{9} / \mathrm{L}$, and Group 3 (No lymphopenia), patients with lymphocyte counts $\geq 1.5 \times$ $10^{9} / \mathrm{L}$. The disease was then subdivided into classes according to disease severity, in accordance with the guidelines issued by the National institute of Health Pakistan. Outcomes were divided into Alive, Dead \& Unknown (when patients left against medical advice). The three groups were then compared with each other for age, gender, disease severity \& outcomes to discern a link. All information including demographics, disease severity, laboratory findings \& clinical outcomes were first noted down in a predesigned Performa, later entered and analyzed on SPSS version 22. For quantitative variables such as age and lymphocyte counts, the mean and standard deviation was calculated and for qualitative variables like gender, disease severity and outcomes, percentage and frequency were calculated. Chi Square test and ANOVA were applied where ever appropriate. A p value $\leq 0.05$ was taken as statistically significant.

\section{RESULTS}

A total of 216 patients were included in the study. Of these 172 $(79.6 \%)$ were males (male to female ratio of 3.91:1). The mean age was $54.61 \pm 14.35$ years.

Table 1: Demographic data of COVID-19 patients $(n=216)$.

\begin{tabular}{|l|l|c|}
\hline \multicolumn{2}{|c|}{ Characteristics } & Frequency (\%) \\
\hline Mean Age (years) & \multicolumn{1}{|c|}{$54.61 \pm 14.35$} \\
\hline \multirow{4}{*}{ Gender } & Male & $172(79.6)$ \\
\cline { 2 - 3 } & Female & $43(19.9)$ \\
\hline \multirow{4}{*}{ Disease Severity } & Mild & $78(36.1)$ \\
\cline { 2 - 3 } & Moderate & $72(33.3)$ \\
\cline { 2 - 3 } & Severe & $46(21.3)$ \\
\cline { 2 - 3 } & Critical & $20(09.3)$ \\
\hline \multirow{3}{*}{ Outcome } & Alive & $115(53.2)$ \\
\cline { 2 - 3 } & Death & $41(19.0)$ \\
\cline { 2 - 3 } & LAMA/DOW & $19(09.4)$ \\
\hline
\end{tabular}

Classic Lymphopenia was found in 67(31.5\%) patients, 66(31.0\%) had borderline lymphopenia, and 80(37.6\%) had no lymphopenia (Figure 1).

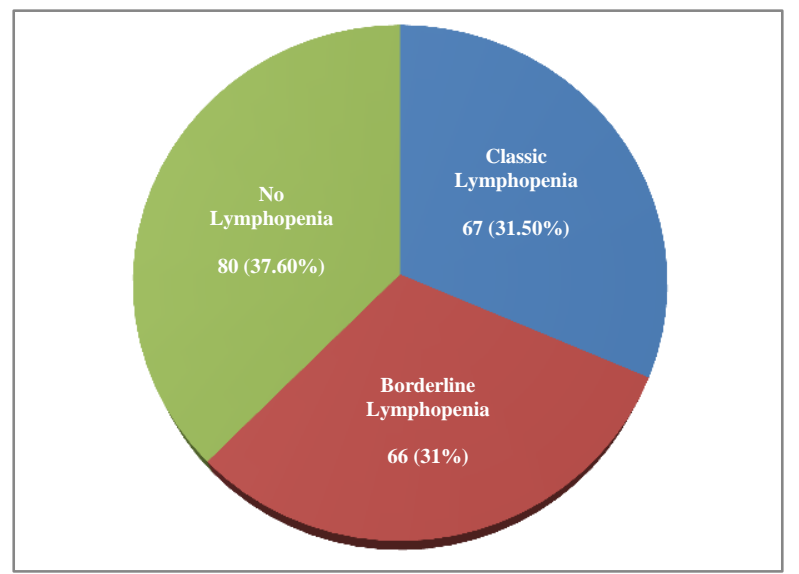

Figure 1: Lymphopenia groups in COVID-19 patients $(n=216)$.

Table 2 shows the disease categories of Lymphopenia groups. In Group 1 patients, 14(20.9\%) had mild disease, 21(31.3\%) had moderate disease, $18(26.9 \%)$ had severe disease and $14(20.9 \%)$ had critical disease. In Group 2 patients, 31(47.0\%) had mild disease, $17(25.8 \%)$ had moderate disease, $14(21.2 \%)$ had severe disease, and 04(06.1\%) had critical disease. In Group 3 patients, $32(40.0 \%)$ had mild, 32(40.0\%) had moderate, 14(17.5\%) had severe, and $02(02.5 \%)$ had critical disease.

Mortality in Group 1 was higher at $37.3 \%(n=25)$ compared to $12.1 \%$ in Group 2 and $10.0 \%$ in Group 3 ( $\mathrm{p}=0.001)$. Gender was found to be statistically linked to lymphopenia with male 
prevalence seen at $91 \%(\mathrm{n}=61)$ for Group 1 and $80.3 \%(\mathrm{n}=53)$ for Group 2. However no statistically significant link could be established between age and lymphopenia. Severe / critical disease and adverse outcome had a statistically significant association with lymphopenia (Table 2).

Table 2: Comparison of characteristics between the three groups $(n=216)$.

\begin{tabular}{|c|c|c|c|c|c|}
\hline \multicolumn{2}{|c|}{ Characteristics } & $\begin{array}{c}\text { Group I ( } \mathrm{n}=67) \\
\text { (Classic lymphopenia) }\end{array}$ & $\begin{array}{c}\text { Group II (n=66) } \\
\text { (Borderline lymphopenia) }\end{array}$ & $\begin{array}{l}\text { Group III (n=80) } \\
\text { (No lymphopenia) }\end{array}$ & p value \\
\hline \multicolumn{2}{|l|}{ Mean Age (Years) } & $57.93 \pm 13.45$ & $53.82 \pm 14.11$ & $52.80 \pm 15.13$ & 0.082 \\
\hline \multirow{2}{*}{ Gender } & Male & $61(91.0)$ & $53(80.3)$ & $56(70.0)$ & \multirow{2}{*}{0.014} \\
\hline & Female & $06(09.0)$ & $12(18.2)$ & $24(30.0)$ & \\
\hline \multirow{4}{*}{ Disease Severity } & Mild & $14(20.9)$ & $31(47.0)$ & $32(40.0)$ & \multirow{4}{*}{$<0.001$} \\
\hline & Moderate & $21(31.3)$ & $17(25.8)$ & $32(40.0)$ & \\
\hline & Severe & $18(26.9)$ & $14(21.2)$ & $14(17.5)$ & \\
\hline & Critical & $14(20.9)$ & $04(06.1)$ & $02(02.5)$ & \\
\hline \multirow{3}{*}{ Outcome } & Alive & $29(43.3)$ & $36(54.4)$ & $48(60.0)$ & \multirow{3}{*}{0.001} \\
\hline & Death & $25(37.3)$ & $08(12.1)$ & $08(10.0)$ & \\
\hline & LAMA/DOW & $08(12.0)$ & $06(09.1)$ & $05(06.3)$ & \\
\hline
\end{tabular}

\section{DISCUSSION}

The dominant gender in this study was male; $79.6 \%$ of the data subset was male. Contrary to our result, initial reports from China and Europe found roughly similar numbers of males and females being affected by COVID-19. ${ }^{11}$ A study from China, made up of 425 patients, found that $56 \%$ of their study population was male. Similarly, another Chinese study, with 140 patients found, that $50.7 \%$ of their patients were males. ${ }^{12}$ Studies from New York and 7 other US states found an equal number of male to female ratio of affected patients whereas the remaining 16 states, including Washington exhibited a male dominance (i.e. male to female ratio 1:0.9/0.8) as was seen in our study. Unusually another study out of a long-term care facility in Washington, with a 167 COVID-19 cases showed the majority of the cases to be women $(68 \%$ of the residents and $76 \%$ of the healthcare workers). ${ }^{11}$

The mean age of our patients was $54.61 \pm 14.35$ years. Two studies reported findings similar to ours i.e. a study from Shanghai, ${ }^{13}$ where the mean age of the patients was 50.1 years and another study from Wuhan with 221 subjects, where the median age was 55.0 years. ${ }^{14}$ Contrary to this, certain other Chinese studies reported their subjects as being in their midforties i.e. 46 years in Hubei and 45 years in Chongqing. ${ }^{15,16}$ American studies reported older patients e.g. a study out of New York comprising of 393 patients, reported a median age of 62.2 years. ${ }^{6}$ Similarly another US study reported a total of 43 patients as having a mean age of 65.5 years. ${ }^{17}$ Studies out of India indicated a younger patient pool e.g. a study with a data set of 147 patients, predominantly male i.e. $70.7 \%$, had a mean age of $40.9,{ }^{18}$ and another Indian study similarly reported a mean age of its study cohort as 40.3 years. ${ }^{19}$

Regarding lymphopenia, $31.5 \%$ of our study subjects were lymphopenic $\left(<1 \times 10^{9} / \mathrm{L}\right), 31.0 \%$ had borderline lymphopenia $\left(1-1.5 \times 10^{9} / \mathrm{L}\right)$ and $37.6 \%$ had no lymphopenia at all on admission. These findings are supported by several studies from around the globe. In a single centre study of COVID-19 patients from Shanghai they found that the most common laboratory abnormality was lymphopenia, $48.5 \%$ of their patients had decreased lymphocytes on admission. ${ }^{9}$ Another study from
Wuhan, with 221 subjects, showed $33.0 \%$ of their patients as having leukopenia and $73.8 \%$ lymphopenia on admission. ${ }^{10}$ Another study from Hubei, with 291 COVID-19 patients, found lymphopenia in $22.7 \%$ of their patients. They reported that patients with severe/critical disease had a relatively lower level of lymphocytes, much like our findings. ${ }^{11}$ Another Chinese study of 51 COVID-19 patients also reported lymphopenia in $51.0 \%$ of their patients. ${ }^{12}$ An American study with 42 diagnosed COVID-19 patients in Des Moines, found lymphopenia in $75 \%$ of their patients. ${ }^{17}$ A study conducted in New York on 393 patients showed that most of their data set i.e. $90 \%$ had lymphopenia. ${ }^{13}$

The present study showed that based on lymphopenia groups of the study and the proportion and severity of disease in each group (Table 2), a statistically significant association was found between lymphopenia and severe / critical disease and mortality.

In Hubei, a double center observational study found that compared to the mild / moderate disease severity group, the severe / critical disease group had a lower level of lymphocytes $(\mathrm{p}<0.01){ }^{15}$

Another study found significant reduction in lymphocyte count in severe vs non-severe COVID-19, so that those with lymphopenia had a 3-fold higher risk of developing severe infection; lymphocyte counts offer a useful, inexpensive and easily accessible biomarker that can predict severity and clinical outcomes. ${ }^{8}$

Another study with 222 patients found that severe cases were more frequently found in patients with higher pro inflammatory cytokines and decreased $\mathrm{T}$ cell counts. ${ }^{20}$ As for mortality, $37.3 \%$ of the lymphopenic patients expired, $43.3 \%$ were alive and the remainder had an unknown outcome as they left against medical advice and were not followed thereafter.

In a study conducted in Wuhan, $19.0 \%$ patients were discharged and $5.4 \%$ patients died; $80 \%$ of severe cases received ICU care, with $52.3 \%$ eventually being discharged after clinical improvement and $20.5 \%$ ended up dying. ${ }^{14}$ In another study of 
393 COVID-19 patients, 90\% were lymphopenic, and of these $40(10.2 \%)$ patients, died, 260(66.2\%) were discharged from the hospital, and outcome data are incomplete for the remaining 93(23.7\%) patients. ${ }^{6}$ This result varies from our findings.

A study of 17 death cases, 7 severe cases and 11 moderate cases of COVID-19 found that the most significant and consistent hematological correlation with severity, disease progression and adverse outcomes was lymphopenia. In conclusion they discerned that lymphopenia was an effective and reliable indicator of severity and adverse outcomes. ${ }^{7}$

Patients admitted to the ICU in Wuhan showed leucopenia and lymphopenia as compared to non-ICU patients on admission, indicating that this can be a prospective marker of severity \& sequelae. ${ }^{8}$ Recent articles reported $85 \%$ of critically ill patients with COVID-19 showed lymphopenia as a hallmark of COVID19 ICU and deceased patients. ${ }^{21} \mathrm{~A}$ meta-analysis identified a number of possible variables that can serve as clinical predictors of severe and fatal COVID-19. This study recommended closely monitoring of WBC and lymphocyte counts in hospitalized COVID-19 patients with respiratory complications as these laboratory aberrations were commonly observed in nonsurvivors and could predict a potential progression to critical illness. ${ }^{22}$ Another study found that among hematological indices, lymphopenia was clearly associated with severe disease and non-survivors had significantly lower lymphocyte counts than survivors. ${ }^{23}$ The differences in age, gender and outcomes between our studies and others can be explained by a difference in the general quality of life, easy availability and access to medical and healthcare services, and a difference in social, cultural and territorial norms and practices.

\section{CONCLUSION}

A significant association was found between lymphopenia at admission and severe / critical disease and adverse sequelae. Thus a lymphocyte count can be a reliable indicator that the patient requires urgent and rigorous medical attention and hospital admission. This could significantly reduce mortality and morbidity in low to middle income countries such as Pakistan where healthcare resources are limited, by streamlining potential critical patients.

\section{REFERENCES}

1. Rothan HA, Byrareddy SN. The epidemiology and pathogenesis of coronavirus disease (COVID-19) outbreak. J Autoimmun. 2020May;109:102433.

2. Zhu N, Zhang D, Wang W, Li X, Yang $\mathrm{B}$, Song J, et al. A novel coronavirus from patients with pneumonia in China, 2019. New Engl J Med. 2020 Feb 20;382(8):727-33.

3. Novel coronavirus (2019-nCov) situation reports. [cited 2020 August 22]. Available from: https://www.who.int/iemergencies/diseas es/novel-coronavirus-2019/situationreport.

4. Wang D, Hu B, Hu C, Zhu F, Liu X, Zhang J, et al. Clinical characteristics of 138 hospitalized patients with 2019 novel coronavirus-infected pneumonia in Wuhan, China. JAMA. 2020 Mar 17;323(11):1061-9ss.

5. Guan WJ, Ni ZY, Hu Y, Liang WH, Ou $\mathrm{CQ}, \mathrm{He} \mathrm{JX}$, et al. Clinical characteristics of coronavirus disease 2019 in China. New Engl J Med. 2020;382(18):1708-20.

6. Goyal P, Choi JJ, Pinheiro LCT, Schenck EJ, Chen R, Jabri A, et al. Clinical characteristics of Covid-19 in New York City. N Engl J Med. 2020;382(24):23724.

7. Tan L, Wang Q, Zhang D, Ding J, Huang Q, Tang Y-Q, et al. Lymphopenia predicts disease severity of COVID-19:a descriptive and predictive study. Sig Transduct Target Ther. 2020 Mar 27;(1):1-3
8. Zhao Q, Meng M, Kumar R, Wu Y, Huang J, Deng Y, et al. Lymphopenia is associated with severe coronavirus disease 2019 (COVID-19) infections: A systemic review and meta-analysis. Int $\mathbf{J}$ Infect Dis. 2020;96:131-5.

9. Wang W, Xu Y, Gao R, Lu R, Han K, $\mathrm{Wu}$ G, et al. Detection of SARS-CoV-2 in different types of clinical specimens. JAMA. 2020 May 12; 323(18):1843-4.

10. Xie X, Zhong Z, Zhao W, Zheng C,Wang F, Liu J. Chest CT for typical 2019-nCov pneumonia relationship to negative RT- PCR testing. Radiology. 2020 Aug;296(2):E41-E45.

11. Klein S, Dhakal S, Ursin R, Deshpante S, Sandberg K, Mauvais-Jarvis F. Biological sex impacts COVID-19 outcomes.PLoS Pathog. 2020;16:e 1008570 .

12. Jin JM, Bai P, He W, Wu F, Liu XF, Han $\mathrm{DM}$, et al. Gender differences in patients with COVID-19: focus on severity and mortality. Front Public Health. 2020;8:152.

13. Cao M, Zhang D, Wang Y, Lu Y, Zhu X, Li Y, et al. Clinical features of patients infected with the 2019 novel coronavirus (COVID-19) in Shanghai, China. medRxiv. Preprint. 2020 Mar 6.

14. Zhang G, Hu C, Luo L, Fang F, Chen Y, $\mathrm{Li}$ J, et al. Clinical features and outcomes of 221 patients with COVID-19 in Wuhan, China. J Clin Virol. 2020 Jun; 127:104364.

15. Chen X, Zheng F, Qing Y, Ding S, Yang $\mathrm{D}$, Lei $\mathrm{C}$, et al. Epidemiological and clinical features of 291 cases with coronavirus disease 2019 in areas adjacent to Hubei, China: a double-center observational study. MedRxiv 2020 Jan 1.

16. Liu L, Gao J-Y, Hu W-E, Zhang X$\mathrm{X}$, Guo L, Liu C-Q, et al. Clinical characteristics of 51 patients discharged from hospital with COVID-19 in Chongqing, China. MedRxiv. 2020. doi:https://doi.org/10.1101/2020.02.20.2 0025536.

17. Aggarwal S, Garcia-Telles N, Aggarwal G, Lavie C, Lippi G, Henry BM. Clinical features, laboratory characteristics, and outcomes of patients hospitalized with coronavirus disease 2019 (COVID-19): Early report from the United States. Diagnosis (Berl). 2020 May 26;7(2):916.

18. Parry AH, Wani AH, Yaseen M, Dar KA, Choh NA, Khan NA, et al. Spectrum of chest computed tomographic (CT) findings in coronavirus disease-19 (COVID-19) patients in India. Eur J Radiol. 2020 Aug;129:109147.

19. Gupta N, Agrawal S, Ish P, Mishra S, Gaind R, Usha G, et al. Clinical and epidemiologic profile of the initial COVID-19 patients at a tertiary care centre in India. Monaldi Arch Chest Dis. 2020 Apr 10;90(1).

20. Zhang B, Zhou X, Zhu C, Feng F, Qiu Y, Feng $\mathbf{J}$, et al. Immune phenotyping based on neutrophil to lymphocyte ratio and IgG predicts disease severity and outcome for patients with COVID-19. medRxiv.2020.doi:https://doi.org/10.110 1/2020.03.12.20035048. 
21. Bermejo-Martin JF, Almansa R, Menendez R, Mendez R, Kelvin DJ, Torres A. Lymphopenic community acquired pneumonia as signature of severe COVID-19 infection. J Infect. 2020;80(5):e23-e4.
22. Henry BM, de Oliveira MHS, Benoit S, Plebani M, Lippi G. Hematologic, biochemical and immune biomarker abnormalities associated with severe illness and mortality in coronavirus disease 2019 (COVID-19): a meta- analysis. Clin Chem Lab Med. 2020;58(7):1021-8.

23. Velavan TP, Meyer CG. Mild versus severe COVID-19: Laboratory markers. Int Journal Infect Dis. 2020;95:304-7. 